\title{
Throughput Optimization of Broadcasting Protocol in Vehicular Ad hoc Networks
}

\author{
LIU Hongfei ${ }^{1,2, a}$, LI Lljun ${ }^{3, b}$ and Liu ZHendong ${ }^{1, c}$ \\ ${ }^{1}$ Chongqing Creation Vocational College, Chongqing, 402160, China \\ ${ }^{2}$ College of Mechanical Engineering Chongqing University, Chongqing, 400044, China \\ ${ }^{3}$ Chongqing Institute of Technology, School of Mathematics and Sciences,400050, Chongqing, \\ China \\ acqtxxy123@126.com, ํllj.liu@cqut.edu.cn, cliuzhendong@126.com
}

\begin{abstract}
Keywords: Vehicular Ad hoc Networks; broadcasting protocol; saturated throughput; Scalability Abstract. It is important to maximize the saturated throughput of VANET broadcasting protocol for fully utilizing wireless channel. The saturated throughput of broadcasting protocol is related to contention windows valve and contending nodes in the networks. We propose a discrete markov analytic model for performance evaluation broadcasting protocol under saturation condition for VANET, and analysis the relationship between the number of vehicle nodes and the contention windows. So, the analysis results show suitable contention window value can optimize the throughput of broadcasting protocol of VANET. The results show that the proposed analytic model and optimization contention windows value can enhance the system throughput.
\end{abstract}

\section{Introduction}

Vehicular Ad-Hoc Networks (VANET) are wireless networks that does not require any fixed infrastructure, concerning the traffic safety applications warning messages have to be quickly and smartly to be disseminated in order to reduce the required dissemination time and to increase the number of vehicles receiving the traffic information[1,2]. One of the most important factors that would make it possible to reach this goal is the design of effective broadcast protocols. A large portion of the messages sent in a vehicular network will be broadcast messags. Some of the uses for broadcast messages are: sending emergency warning messages, periodically broadcasting a vehicles state, etc.

However, since there is no MAC layer acknowledgement, retransmission and recovery on broadcast frames within an 802.11 based VANET, the reliability, system throughput and reception rates of broadcast messages could be very low, especially under saturation conditions. Furthermore, the broadcasting of warning messages raises a number of challenging issues.

In this paper, we mainly focus on performance analysis of IEEE 802.11 Broadcasting protocol. In VNAET, emergency warning messages broadcasting performance are influenced by many factors. In this report, we make an attempt to analytically evaluate the performance of broadcasting under saturated conditions.

\section{Channel Performance}

We can easily infer from the analytical Markov Chain Model of the IEEE802.11 broadcasting protocol

$$
b_{0}=\frac{2(1-p)}{W_{0}+1-2 p}
$$

As any transmission occurs when the backoff counter value is equal to zero, it is evident that

$$
b_{0}=\tau=\frac{2(1-p)}{W_{0}+1-2 p}
$$


In equation (2), $b_{0}$ depends on the probability $\mathrm{p}$, which is still unknown. For an ideal channel, the probability that the channel being busy when a node is trying decrement its backoff counter is the probability that among the rest of the nodes, at least one is in transmission state. Therefore,

$$
p=1-p_{i}=1-\frac{1}{1-\tau}\left(e^{-2 \gamma R \tau}-e^{-2 \gamma R}\right)
$$

The transmission probability $\tau$ can be obtained simultaneously. In the remainder of this section, we will derive closed form expressions for broadcast reliability and saturation throughput in terms of the $\tau$.

\section{Saturation Throughput Analysis}

Where $\sigma$ is an empty slot time. For the time intervals $T_{s}, T_{c}$, we follow the definition given by[]

$$
T=T_{s}=T_{c}=T_{M A C}+T_{P H Y}+\frac{E[p k g]}{t r}+D I F S+\delta
$$

Related values and description of parameters are summarized in Table1. The normalized throughput $S$ is defined as:

$$
s=\frac{p_{t r} p_{s} T_{p l}}{\left(1-p_{t r}\right) \sigma+p_{t r} p_{s} T_{s}+p_{t r}\left(1-p_{s}\right) T_{s}}=\frac{p_{t r} p_{s} T_{p l}}{\left(1-p_{t r}\right) \sigma+p_{t r} T_{s}}
$$

From equation (5), we can see that existence of the appropriate window raises an optimal throughput for the number of network nodes, IEEE802.11 broadcasting protocol would need to operate under window sizes much lager than the values, selecting a window lager than the optimal value would prevent the networks from achieving the maximum throughput.

Since $T_{p l}, T$ are constants, the normalized throughput becomes a function of the transmission probability $\tau$. Rearranging Equation (3), we obtain:

$$
s=T_{p l} /\left\{\left(\sigma\left(\left(1-p_{t}\right) / p_{t}+T_{s}\right) / p_{s}\right\}\right.
$$

Base on idea of literature [3], we suppose $k=\sqrt{T_{s} /(2 \sigma)}$, and obtain optimal transmission probability $\tau_{\text {opt }}$.

$$
\begin{gathered}
\tau_{\text {opt }}=\frac{\sqrt{\left[n+2(n-1)\left(T_{s} / \sigma-1\right)\right] / n}-1}{(n-1)\left(T_{s} / \sigma-1\right)} \approx \frac{1}{n \sqrt{T_{s} /(2 \sigma)}}=\frac{1}{n k} \\
p=1-(1-\tau)^{n-1}=1-\left(1-\frac{1}{n k}\right)^{n-1}=1-\left(1-\frac{1}{n k}\right)^{n} /\left(1-\frac{1}{n k}\right)
\end{gathered}
$$

we set $p^{*}=\left(1-\frac{1}{n k}\right)^{n}$, then $\left(p^{*}\right)^{k}=\left(1-\frac{1}{n k}\right)^{n k}$

when $n k$ is large enough, then

$$
\left(p^{*}\right)^{k}=\left(1-\frac{1}{n k}\right)^{n k}=\frac{1}{e}, \quad p^{*}=e^{-\frac{1}{k}}
$$

From equation (8) and (9), $p$ can be denoted as

$$
p=1-e^{-\frac{1}{k}} /\left(1-\frac{1}{n k}\right)=1-\frac{1}{e^{1 / k}(1-1 / n k)}
$$

The approximate optimized transmission probability is given as

$$
W_{o p t}=\frac{2 n k-2}{e^{1 / k}(1-1 / n k)}+1
$$




\section{Numerical Results}

In order to validate the proposed model, we compare the reliability, delay and the throughput performance of the network obtained from the simulations and numerical analysis by matlab7.1, respectively. Considering VANET as a typical application of broadcast communication, all broadcast packets have the same size. So, it is 128-byte per packets.

Table 1 Relationship among channel busy probability, $W$ and number of nodes

\begin{tabular}{cccccc} 
& 16 & 32 & 64 & 128 & 256 \\
\hline 5 & 0.3001 & 0.1853 & 0.1059 & 0.0572 & 0.0299 \\
10 & 0.4632 & 0.3204 & 0.2017 & 0.1169 & 0.0637 \\
15 & 0.5543 & 0.4082 & 0.2733 & 0.1668 & 0.0943 \\
20 & 0.6143 & 0.4713 & 0.3297 & 0.2095 & 0.1222 \\
25 & 0.6575 & 0.5194 & 0.3756 & 0.2465 & 0.1478 \\
30 & 0.6905 & 0.5577 & 0.4140 & 0.2792 & 0.1713 \\
35 & 0.7166 & 0.5891 & 0.4468 & 0.3082 & 0.1931 \\
40 & 0.7380 & 0.6155 & 0.4751 & 0.3342 & 0.2133 \\
45 & 0.7558 & 0.6379 & 0.4999 & 0.3578 & 0.2322 \\
50 & 0.7710 & 0.6574 & 0.5220 & 0.3792 & 0.2498 \\
55 & 0.7841 & 0.6745 & 0.5416 & 0.3988 & 0.2664 \\
60 & 0.7955 & 0.6896 & 0.5594 & 0.4169 & 0.2820 \\
\hline
\end{tabular}

Table.1 shows relationship among channel busy probability, $W$ and number of nodes. The saturation throughput results over the number of nodes with different contention window sizes. As we see from Fig. 1 that the analytical model highly matches the simulation results. For a given the number of nodes in transmission range, there exists an optimum of contention windows for which the broadcast throughput is maximized.

Table 2. Analysis and comparison of optimal and non-optimal throughput

\begin{tabular}{cccc} 
Nodes & non-optimal throughput $(W=128)$ & optimal throughput & $(\%)$ \\
\hline 5 & 0.6072 & 0.6400 & 3.28 \\
10 & 0.6386 & 0.6399 & 0.13 \\
15 & 0.6383 & 0.6399 & 0.16 \\
20 & 0.6309 & 0.6396 & 0.87 \\
25 & 0.6213 & 0.6399 & 2.86 \\
30 & 0.6110 & 0.6399 & 3.95 \\
35 & 0.6004 & 0.6399 & 4.96 \\
40 & 0.5903 & 0.6399 & 5.87 \\
45 & 0.5812 & 0.6399 & 6.84 \\
50 & 0.5716 & 0.6400 & 7.72 \\
55 & 0.5628 & 0.6400 & 8.50 \\
\hline 60 & 0.5548 & 0.6398 & \\
\hline
\end{tabular}


Table. 2 shows the broadcasting delay over the number of nodes with different contention window sizes. We can see that under high offered traffic, the biggest delay is $45 \mathrm{~ms}$ which is much less than the message life time 500ms. Comparing the obtained performance with requirements set for safety related VANETS communication, it is no problem for packet delivery delay to meet the requirement.

\section{conclusion}

In this paper, we develop an analytical model for IEEE 802.11 broadcast to derive closed form expressions of the saturation throughput, the saturation delay, and the packet reception ratio. Several important performance indices are derived from the proposed analytical model taking IEEE 802.11 standard MAC-broadcasting protocol and saturation traffic into account.

Our analysis reveals that relatively big backoff window size and short message length help improve reliability of the broadcast communication. From the above observations, it may appear that, for a given broadcast network, any arbitrarily high broadcast reliability can be achieved by simply selecting a large enough contention window. However, as we shall see in the next section, scaling up the contention window may negatively impact the system throughput, leading to the so called reliability-throughput tradeoff.

\section{References}

[1] Renukadevi M, Balasubramanian C, Angelin Nithya Devi M A reliable application level broadcasting protocol for VANET. 2015 International Conference on Soft-Computing and Networks Security (ICSNS).

[2] Yizhi Wang,Yi Zhang, Jianming Hu, Xin Pei.An Analysis on Stochastic Broadcast Range in VANET Based on Generalized Packet Loss Model.2015 IEEE 18th International Conference on Intelligent Transportation Systems (ITSC).

[3] Saleh Y, Mahmoud SM, Mahmood F. Vehicular Ad Hoc Networks (VANETs): Challenges and Perspectives[C]. 2006 6th International Conference on ITS Telecommunications Proceedings, 2006. 761-766.

[4] Zhang JQ, Liu KH, Shen XM. A Novel Overlay Token Ring Protocol for Inter-Vehicle Communication[C]. IEEE International Conference on Communications, (ICC08)2008. 4904-4909.

[5] Ravi M, Yadumurthy A, Chimalakonda M. Reliable MAC broadcast protocol in directional and omni-directional transmissions for vehicular ad hoc networks[C]. Proceedings of the 2nd ACM international workshop on Vehicular ad hoc networks,(VANET2005). 10-19.

[6] Ma XM, Chen XB. Saturation Performance of IEEE 802.11 Broadcast Networks [J]. IEEE Communications Letters, 2007. On page(s): 686-688.

[7] Chen XB, Refai HH. Ma XM. Saturation Performance of IEEE 802.11 Broadcast Schemes in Ad Hoc Wireless LANs[C]. IEEE 66th Vehicular Technology Conference, 2007. VTC-2007 Fall, On page(s): 1897-1901.

[8] Wang Z, Mahbub H. Analytical Evaluation of the 802.11 Wireless Broadcast under Saturated Conditions. ftp://ftp.cse.unsw.edu.au/pub/doc/papers/UNSW/0801.pdf. 2008.

[9] Cheng ST, Wu MZ. Performance evaluation of ad-hoc WLAN by M/G/1 queueing model. International Conference on Information Technology: Coding and Computing, 2005. ITCC 2005. Page(s): 681- 686.

[10] The ns-2 Network Simulator. http://www.isi.edu/nsnam/ns/. 